\title{
BINARY CONSTRAINED FLOWS AND SEPARATION OF VARIABLES FOR SOLITON EQUATIONS
}

\author{
WEN-XIU MA ${ }^{1}$ and YUNBO ZENG ${ }^{2}$
}

(Received 1 May, 2000)

\begin{abstract}
In contrast to mono-constrained flows with $N$ degrees of freedom, binary constrained flows of soliton equations, admitting $2 \times 2$ Lax matrices, have $2 N$ degrees of freedom. Currently existing methods only enable Lax matrices to yield the first $N$ pairs of canonical separated variables. An approach for constructing the second $N$ pairs of canonical separated variables with $N$ additional separated equations is introduced. The Jacobi inversion problems for binary constrained flows are then established. Finally, the separability of binary constrained flows together with the factorization of soliton equations by the spatial and temporal binary constrained flows leads to the Jacobi inversion problems for soliton equations.
\end{abstract}

\section{Introduction}

Separation of variables is one of the most universal methods for solving completely integrable models, both classical and quantum. If a finite-dimensional integrable Hamiltonian system (FDIHS) with $m$ degrees of freedom has $m$ functionally independent and involutive integrals of motion $P_{i}, 1 \leq i \leq m$, separation of variables $[7,14]$ leads to the construction of $m$ pairs of canonical variables

$$
\left\{u_{k}, u_{l}\right\}=\left\{v_{k}, v_{l}\right\}=0, \quad\left\{v_{k}, u_{l}\right\}=\delta_{k l}, \quad 1 \leq k, l \leq m,
$$

and $m$ separated equations

$$
f_{k}\left(u_{k}, v_{k}, P_{1}, \ldots, P_{m}\right)=0, \quad 1 \leq k \leq m .
$$

Such pairs are called canonical separated variables.

\footnotetext{
'Department of Mathematics, University of South Florida, Tampa, FL 33620-5700, USA; e-mail: mawx@math.usf.edu.

${ }^{2}$ Department of Mathematical Sciences, Tsinghua University, Beijing 100084, China;

e-mail: yzeng@tsinghua.edu.cn.

(C) Australian Mathematical Society 2002, Serial-fee code 1446-1811/02
} 
For a FDIHS admitting a $2 \times 2$ Lax matrix, there exists a general method for constructing canonical separated variables based on the Lax matrix (for example, see $[4-7,14-16])$. The corresponding separated equations enable us to express the generating function of canonical transformation in a completely separated form as an Abelian integral on the associated invariant spectral curve. The resulting linearizing map is essentially the Abel map to the Jacobi variety of the spectral curve, thereby providing a link with the algebra-geometric linearization method [3]. An important feature of the separation of variables for a FDIHS is that the number of pairs of canonical separated variables must be equal to the number of degrees of freedom. However, in some cases, it is found that the existing method may not yield enough pairs of canonical separated variables. It is a challenging problem [14] to construct the additional canonical separated variables which are required for separation of variables.

Binary constrained flows of soliton hierarchies, which have recently attracted a great deal of attention (for example, see [9,11-13]), are cases of this type, and need to be handled by a different approach. Binary constrained flows admitting $2 \times 2$ Lax matrices have $2 N$ degrees of freedom. By using existing methods [7, 14], the Lax matrices allow us to directly construct the first $N$ pairs of canonical separated variables $u_{1}, \ldots, u_{N}$ and $v_{1}, \ldots, v_{N}$. In this paper, we show an approach for determining the second $N$ pairs of canonical separated variables and $N$ additional separated equations for binary constrained flows. The crucial point is to construct a new set of generating functions $\widetilde{B}(\lambda)$ and $\widetilde{A}(\lambda)$ defining $u_{N+1}, \ldots, u_{2 N}$ by the set of zeros of $\widetilde{B}(\lambda)$ and $v_{N+k}=\widetilde{A}\left(u_{N+k}\right), 1 \leq k \leq N$. To keep the canonical conditions (1.1) and obtain the separated equations (1.2), it is found that certain commutator relations need to be imposed on $\widetilde{B}(\lambda)$ and $\widetilde{A}(\lambda)$, and that $\widetilde{A}(\lambda)$ has some link with the common generating function of integrals of motion of binary constrained flows, which also provides a clue for constructing $\widetilde{B}(\lambda)$ and $\widetilde{A}(\lambda)$. Having analyzed the separation of variables, the Jacobi inversion problems can be naturally presented for binary constrained flows.

Separation of variables for soliton equations consists of two steps [16]. The first step is to factorize 1+1-dimensional soliton equations into two commuting spatial and temporal FDIHSs resulting from the spatial and temporal binary constrained flows. The second step is to analyze the separation of variables for these flows to produce their Jacobi inversion problems. Finally, combining the factorization of soliton equations with the Jacobi inversion problems for the spatial and temporal binary constrained flows enables us to establish the Jacobi inversion problems for soliton equations. We will use the AKNS equations [1] to illustrate the whole process. Of course, the approach adopted can be applied to the whole AKNS hierarchy and other soliton hierarchies. 


\section{Separation of variables for binary constrained flows}

Let us first describe binary constrained flows admitting $2 \times 2$ Lax matrices and then show an approach for constructing $2 N$ pairs of canonical separated variables.

Assume that a soliton hierarchy

$$
u_{t_{n}}=K_{n}(u)=J \frac{\delta \tilde{H}_{n}}{\delta u}, \quad u=\left(u_{1}, \ldots, u_{q}\right)^{T}, \quad n \geq 0,
$$

where $J$ is a Hamiltonian operator, is determined by a spectral problem

$$
\phi_{x}=U \phi=U(u, \lambda) \phi, \quad U=\left(U_{i j}\right)_{2 \times 2}, \quad \phi=\left(\phi_{1}, \phi_{2}\right)^{T},
$$

and the associated spectral problems

$$
\phi_{l_{n}}=V^{(n)} \phi=V^{(n)}\left(u, u_{x}, \ldots ; \lambda\right) \phi, \quad V^{(n)}=\left(V_{i j}^{(n)}\right)_{2 \times 2} .
$$

The compatibility conditions of the adjoint spectral problem

$$
\psi_{x}=-U^{T}(u, \lambda) \psi, \quad \psi=\left(\psi_{1}, \psi_{2}\right)^{T}
$$

and the adjoint associated spectral problems $\psi_{t_{n}}=-V^{(n) T} \psi=-V^{(n) T}\left(u, u_{x}, \ldots ; \lambda\right) \psi$ still give rise to the same soliton hierarchy (2.1).

Upon introducing $N$ distinct eigenvalues $\lambda_{1}, \ldots, \lambda_{N}$, we have the spatial system

$$
\phi_{x}^{(j)}=U\left(u, \lambda_{j}\right) \phi^{(j)}, \quad \psi_{x}^{(j)}=-U^{T}\left(u, \lambda_{j}\right) \psi^{(j)},
$$

where $\phi^{(j)}=\left(\phi_{1 j}, \phi_{2 j}\right)^{T}, \psi^{(j)}=\left(\psi_{1 j}, \psi_{2 j}\right)^{T}, 1 \leq j \leq N$, and the temporal system

$$
\phi_{t}^{(j)}=V^{(n)}\left(u, \lambda_{j}\right) \phi^{(j)}, \quad \psi_{t}^{(j)}=-V^{(n) T}\left(u, \lambda_{j}\right) \psi^{(j)}
$$

where $1 \leq j \leq N$. Let us take the Bargmann symmetry constraint

$$
K_{0}=J \sum_{j=1}^{N} E_{j} J \frac{\delta \lambda_{j}}{\delta u}=J \sum_{j=1}^{N} \psi^{(j) T} \frac{\partial U\left(u, \lambda_{j}\right)}{\partial u} \phi^{(j)},
$$

where $E_{j}$ are normalized constants, and suppose that (2.6) has an inverse function

$$
u=f\left(\xi_{1}, \ldots, \xi_{q}\right), \quad \xi_{i}=\sum_{j=1}^{N} \psi^{(j) T} \frac{\partial U\left(u, \lambda_{j}\right)}{\partial u_{i}} \phi^{(j)}, \quad 1 \leq i \leq q .
$$

Replacing $u$ with $f$ in $N$ replicas of (2.4) and (2.5), we obtain the so-called spatial constrained flow

$$
\phi_{x}^{(j)}=U\left(f, \lambda_{j}\right) \phi^{(j)}, \quad \psi_{x}^{(j)}=-U^{T}\left(f, \lambda_{j}\right) \psi^{(j)},
$$


and the so-called temporal constrained flow

$$
\phi_{t}^{(j)}=V^{(n)}\left(f, f_{x}, \ldots ; \lambda_{j}\right) \phi^{(j)}, \quad \psi_{t}^{(j)}=-V^{(n) T}\left(f, f_{x}, \ldots ; \lambda_{j}\right) \psi^{(j)},
$$

where $1 \leq j \leq N$. Now if $\phi_{i j}$ and $\psi_{i j}$ solve two constrained flows, then $u=$ $f\left(\xi_{1}, \ldots, \xi_{q}\right)$ gives rise to a solution to the soliton equation $u_{t_{n}}=K_{n}(u)$. The above manipulation is called binary nonlinearization $[10,13]$.

It is known that constrained flows (CFs) have natural Lax matrices generated from a solution $M(\lambda)=\left(\begin{array}{cc}A(\lambda) & B(\lambda) \\ C(\lambda) & -A(\lambda)\end{array}\right)$ to $M_{x}=[U, M]$ and $M_{t_{n}}=\left[V^{(n)}, M\right]$ (for example, see $[2,17])$. To determine $2 N$ pairs of canonical separated variables for binary CFs, based on Lax matrices $M(\lambda)$, we search for two sets of generating functions $\bar{A}(\lambda), \bar{B}(\lambda)$ and $\tilde{A}(\lambda), \tilde{B}(\lambda)$ such that

$$
\left\{\begin{array}{l}
\{\bar{B}(\lambda), \bar{B}(\mu)\}=\{\widetilde{B}(\lambda), \widetilde{B}(\mu)\}=\{\bar{A}(\lambda), \bar{A}(\mu)\}=\{\widetilde{A}(\lambda), \widetilde{A}(\mu)\}=0, \\
\{\bar{B}(\lambda), \widetilde{B}(\mu)\}=\{\bar{B}(\lambda), \widetilde{A}(\mu)\}=\{\widetilde{B}(\lambda), \bar{A}(\mu)\}=\{\bar{A}(\lambda), \widetilde{A}(\mu)\}=0, \\
\{\bar{A}(\lambda), \bar{B}(\mu)\}=\frac{\bar{B}(\mu)-\bar{B}(\lambda)}{\lambda-\mu}, \quad\{\widetilde{A}(\lambda), \widetilde{B}(\mu)\}=\frac{\widetilde{B}(\mu)-\widetilde{B}(\lambda)}{\lambda-\mu},
\end{array}\right.
$$

under the standard Poisson bracket

$$
\{F, G\}=\sum_{i=1}^{2} \sum_{j=1}^{N}\left(\frac{\partial F}{\partial \psi_{i j}} \frac{\partial G}{\partial \phi_{i j}}-\frac{\partial F}{\partial \phi_{i j}} \frac{\partial G}{\partial \psi_{i j}}\right) .
$$

Two such sets of generating functions can be constructed from Lax matrices $M(\lambda)$ and a common generating function of integrals of motion for binary CFs. We expect each pair of generating functions to yield $N$ pairs of canonical separated variables, through defining $u_{1}, \ldots, u_{N}$ by the set of zeros of $\bar{B}(\lambda), u_{N+1}, \ldots, u_{2 N}$ by the set of zeros of $\widetilde{B}(\lambda)$, and

$$
v_{k}=\bar{A}\left(u_{k}\right), \quad v_{N+k}=\widetilde{A}\left(u_{N+k}\right), \quad 1 \leq k \leq N,
$$

which will also give us all $2 N$ separated equations. Therefore the separation of variables for binary CFs becomes the problem of finding two sets of generating functions satisfying the above commutator relations (2.9). The whole process will be illustrated by the AKNS equations.

\section{Binary constrained flows of the AKNS equations}

Let us start from the AKNS spectral problem

$$
\phi_{x}=U \phi=U(u, \lambda) \phi, U=\left(\begin{array}{cc}
-\lambda & q \\
r & \lambda
\end{array}\right), \quad \phi=\left(\begin{array}{l}
\phi_{1} \\
\phi_{2}
\end{array}\right), \quad u=\left(\begin{array}{l}
q \\
r
\end{array}\right)
$$


and take the associated spectral problems

$$
\phi_{t_{n}}=V^{(n)} \phi=V^{(n)}(u, \lambda) \phi, \quad V^{(n)}=\sum_{i=0}^{n}\left(\begin{array}{cc}
a_{i} & b_{i} \\
c_{i} & -a_{i}
\end{array}\right) \lambda^{n-i},
$$

with $a_{i}, b_{i}, c_{i}$ being defined by $a_{0}=-1, b_{0}=c_{0}=0, a_{1}=0, b_{1}=q, c_{1}=r$, $a_{2}=q r / 2, \ldots,\left(\begin{array}{l}c_{k+1} \\ b_{k+1}\end{array}\right)=L\left(\begin{array}{l}c_{k} \\ b_{k}\end{array}\right), a_{k+1}=\partial^{-1}\left(q c_{k+1}-r b_{k+1}\right), k \geq 1$, where $L$ is given by $L=\frac{1}{2}\left(\begin{array}{cc}\partial-2 r \partial^{-1} q & 2 r \partial^{-1} r \\ -2 q \partial^{-1} q & -\partial+2 q \partial^{-1} r\end{array}\right)$. The compatibility conditions of (3.1) and (3.2) give the AKNS hierarchy

$$
u_{t_{n}}=J\left(\begin{array}{l}
c_{n+1} \\
b_{n+1}
\end{array}\right)=J \frac{\delta \tilde{H}_{n+1}}{\delta u}, \quad J=\left(\begin{array}{cc}
0 & -2 \\
2 & 0
\end{array}\right), \quad \tilde{H}_{n}=\int \frac{2 a_{n+1}}{n+1} d x, n \geq 1,
$$

which contains the AKNS equations

$$
q_{t_{2}}=-q_{x x} / 2+q^{2} r, \quad r_{t_{2}}=r_{x x} / 2-r^{2} q .
$$

Introducing $N$ distinct eigenvalues $\lambda_{j}, 1 \leq j \leq N$, we have

$$
\begin{array}{ll}
\Phi_{1 x}=-\Lambda \Phi_{1}+q \Phi_{2}, & \Phi_{2 x}=r \Phi_{1}+\Lambda \Phi_{2}, \\
\Psi_{1 x}=\Lambda \Psi_{1}-r \Psi_{2}, & \Psi_{2 x}=-q \Psi_{1}-\Lambda \Psi_{2},
\end{array}
$$

and the Bargmann symmetry constraint reads as

$$
\frac{\delta H_{1}}{\delta u}-\sum_{j=1}^{N} \frac{\delta \lambda_{j}}{\delta u}=\left(\begin{array}{l}
r \\
q
\end{array}\right)-\left(\begin{array}{c}
\left\langle\Psi_{1}, \Phi_{2}\right\rangle \\
\left\langle\Psi_{2}, \Phi_{1}\right\rangle
\end{array}\right)=0
$$

where $\langle\cdot, \cdot\rangle$ denotes the standard inner product of $\mathbb{R}^{N}$ and $\Phi_{i}=\left(\phi_{i 1}, \ldots, \phi_{i N}\right)^{T}$, $\Psi_{i}=\left(\psi_{i 1}, \ldots, \psi_{i N}\right)^{T}, i=1,2, \Lambda=\operatorname{diag}\left(\lambda_{1}, \ldots, \lambda_{N}\right)$. Therefore the spatial constrained flow (2.7) is the following $x$-FDIHS [13]

$$
\Phi_{1 x}=\frac{\partial F_{1}}{\partial \Psi_{1}}, \quad \Phi_{2 x}=\frac{\partial F_{1}}{\partial \Psi_{2}}, \quad \Psi_{1 x}=-\frac{\partial F_{1}}{\partial \Phi_{1}}, \quad \Psi_{2 x}=-\frac{\partial F_{1}}{\partial \Phi_{2}},
$$

with the Hamiltonian $F_{1}=\left\langle\Lambda \Psi_{2}, \Phi_{2}\right\rangle-\left\langle\Lambda \Psi_{1}, \Phi_{1}\right\rangle+\left\langle\Psi_{2}, \Phi_{1}\right\rangle\left\langle\Psi_{1}, \Phi_{2}\right\rangle$. Under the symmetry constraint (3.6) and the $x$-FDIHS (3.7), the binary $t_{2}$-constrained flow (2.8) can be transformed into the following $t_{2}$-FDIHS

$$
\Phi_{1 t_{2}}=\frac{\partial F_{2}}{\partial \Psi_{1}}, \quad \Phi_{2 t_{2}}=\frac{\partial F_{2}}{\partial \Psi_{2}}, \quad \Psi_{1 t_{2}}=-\frac{\partial F_{2}}{\partial \Phi_{1}}, \quad \Psi_{2 t_{2}}=-\frac{\partial F_{2}}{\partial \Phi_{2}},
$$

with the Hamiltonian

$$
\begin{aligned}
F_{2}= & \left\langle\Lambda^{2} \Psi_{2}, \Phi_{2}\right\rangle-\left\langle\Lambda^{2} \Psi_{1}, \Phi_{1}\right\rangle+\left\langle\Psi_{2}, \Phi_{1}\right\rangle\left\langle\Lambda \Psi_{1}, \Phi_{2}\right\rangle \\
& +\left\langle\Lambda \Psi_{2}, \Phi_{1}\right\rangle\left\langle\Psi_{1}, \Phi_{2}\right\rangle-(1 / 2)\left(\left\langle\Psi_{2}, \Phi_{2}\right\rangle-\left\langle\Psi_{1}, \Phi_{1}\right\rangle\right)\left\langle\Psi_{2}, \Phi_{1}\right\rangle\left\langle\Psi_{1}, \Phi_{2}\right\rangle
\end{aligned}
$$


The Lax matrix $M=\left(\begin{array}{cc}A(\lambda) & B(\lambda) \\ C(\lambda) & -A(\lambda)\end{array}\right)$ for the FDIHSs (3.7) and (3.8) is given by [8]

$$
A(\lambda)=-1+\sum_{j=1}^{N} \frac{\psi_{1 j} \phi_{1 j}-\psi_{2 j} \phi_{2 j}}{2\left(\lambda-\lambda_{j}\right)}, \quad B(\lambda)=\sum_{j=1}^{N} \frac{\psi_{2 j} \phi_{1 j}}{\lambda-\lambda_{j}}, \quad C(\lambda)=\sum_{j=1}^{N} \frac{\psi_{1 j} \phi_{2 j}}{\lambda-\lambda_{j}}
$$

A straightforward calculation yields

$$
P(\lambda):=A^{2}(\lambda)+B(\lambda) C(\lambda)=1+\sum_{j=1}^{N}\left[\frac{P_{j}}{\lambda-\lambda_{j}}+\frac{P_{N+j}^{2}}{\left(\lambda-\lambda_{j}\right)^{2}}\right],
$$

where the $P_{j}$ and $P_{N+j}$ are $2 N$ involutive integrals of motion for (3.7) and (3.8),

$$
\begin{aligned}
P_{j}= & \frac{1}{2} \sum_{k \neq j} \frac{1}{\lambda_{j}-\lambda_{k}}\left[\left(\psi_{1 j} \phi_{1 j}-\psi_{2 j} \phi_{2 j}\right)\left(\psi_{1 k} \phi_{1 k}-\psi_{2 k} \phi_{2 k}\right)\right. \\
& \left.+\psi_{2 j} \phi_{2 j}-\psi_{1 j} \phi_{1 j}+4 \psi_{1 j} \phi_{2 j} \psi_{2 k} \phi_{1 k}\right], \quad 1 \leq j \leq N, \\
P_{N+j}= & \frac{1}{2}\left(\psi_{1 j} \phi_{1 j}+\psi_{2 j} \phi_{2 j}\right), \quad 1 \leq j \leq N .
\end{aligned}
$$

It is easy to verify that

$$
\begin{aligned}
& F_{1}=\sum_{j=1}^{N}\left(\lambda_{j} P_{j}+P_{N+j}^{2}\right)-\left(\sum_{j=1}^{N} \frac{P_{j}}{2}\right)^{2}, \\
& F_{2}=\sum_{j=1}^{N}\left(\lambda_{j}^{2} P_{j}+2 \lambda_{j} P_{N+j}^{2}\right)-\left(\sum_{j=1}^{N} \frac{P_{j}}{2}\right) \sum_{j=1}^{N}\left(\lambda_{j} P_{j}+P_{N+j}^{2}\right)+\left(\sum_{j=1}^{N} \frac{P_{j}}{2}\right)^{3} .
\end{aligned}
$$

With respect to the standard Poisson bracket (2.10), it is found [8] that

$$
\begin{aligned}
& \{A(\lambda), A(\mu)\}=\{B(\lambda), B(\mu)\}=\{C(\lambda), C(\mu)\}=0, \\
& \{A(\lambda), B(\mu)\}=\frac{1}{\lambda-\mu}[B(\mu)-B(\lambda)], \\
& \{A(\lambda), C(\mu)\}=\frac{1}{\lambda-\mu}[C(\lambda)-C(\mu)], \\
& \{B(\lambda), C(\mu)\}=\frac{2}{\lambda-\mu}[A(\mu)-A(\lambda)] .
\end{aligned}
$$

Then $\left\{A^{2}(\lambda)+B(\lambda) C(\lambda), A^{2}(\mu)+B(\mu) C(\mu)\right\}=0$ implies that the integrals of motion $P_{j}$ and $P_{N+j}, 1 \leq j \leq N$, are in involution in pairs. The AKNS equations (3.4) are factorized by the $x$-FDIHS (3.7) and the $t_{2}$-FDIHS (3.8). Namely, if $\Phi_{1}, \Phi_{2}, \Psi_{1}$ and $\Psi_{2}$ solve the $x$-FDIHS (3.7) and the $t_{2}$-FDIHS (3.8) simultaneously, then $(q, r)$ given by (3.6) solves the AKNS equations (3.4). 


\section{Separation of variables for the AKNS equations}

The commutator relations (3.11) and a common generating function of integrals of motion

$$
\frac{1}{2} \sum_{j=1}^{N} \frac{\phi_{1 j} \psi_{1 j}+\phi_{2 j} \psi_{2 j}}{\lambda-\lambda_{j}}=\sum_{j=1}^{N} \frac{P_{N+j}}{\lambda-\lambda_{j}}
$$

enable us to construct two sets of generating functions which lead to $2 N$ pairs of canonical separated variables. The required two sets of generating functions for the AKNS equations (3.4) are the following:

$$
\begin{gathered}
\bar{A}(\lambda)=B(\lambda)-A(\lambda)-\tilde{A}(\lambda)=1+\sum_{j=1}^{N} \frac{\left(\psi_{2 j}-\psi_{1 j}\right) \phi_{1 j}}{\lambda-\lambda_{j}} \\
\bar{B}(\lambda)=B(\lambda)-2 A(\lambda)-C(\lambda)=2+\sum_{j=1}^{N} \frac{\left(\psi_{2 j}-\psi_{1 j}\right)\left(\phi_{1 j}+\phi_{2 j}\right)}{\lambda-\lambda_{j}} \\
\widetilde{A}(\lambda)=\frac{1}{2} \sum_{j=1}^{N} \frac{\psi_{1 j} \phi_{1 j}+\psi_{2 j} \phi_{2 j}}{\lambda-\lambda_{j}}, \quad \widetilde{B}(\lambda)=1+\frac{1}{2} \sum_{j=1}^{N} \frac{\left(\phi_{1 j}+\phi_{2 j}\right)^{2}}{\lambda-\lambda_{j}} .
\end{gathered}
$$

Let us now introduce $u_{k}, u_{N+k}, 1 \leq k \leq N$, by

$$
\bar{B}(\lambda)=2 \frac{\bar{R}(\lambda)}{K(\lambda)}, \quad \widetilde{B}(\lambda)=\frac{\widetilde{R}(\lambda)}{K(\lambda)},
$$

where $\bar{R}(\lambda), \widetilde{R}(\lambda)$ and $K(\lambda)$ read as

$$
\bar{R}(\lambda)=\prod_{k=1}^{N}\left(\lambda-u_{k}\right), \quad \widetilde{R}(\lambda)=\prod_{k=1}^{N}\left(\lambda-u_{N+k}\right), \quad K(\lambda)=\prod_{k=1}^{N}\left(\lambda-\lambda_{k}\right) .
$$

A direct computation shows the following result.

THEOREM 4.1. Assume that $\lambda_{j}, \phi_{i j}, \psi_{i j}, i=1,2,1 \leq j \leq N$, are all real, and $u_{1}, \ldots, u_{N}$ are single zeros of $\bar{B}(\lambda)$. Then the variables $u_{1}, \ldots, u_{2 N}$ defined by (4.3) and (4.4), and the variables $v_{1}, \ldots, v_{2 N}$ defined by the corresponding formula (2.11) are canonically conjugated, that is, they satisfy the commutator relations (1.1) with $m=2 N$.

It follows from (4.3) and (4.4) that

$$
\left(\psi_{2 j}-\psi_{1 j}\right)\left(\phi_{1 j}+\phi_{2 j}\right)=2 \frac{\bar{R}\left(\lambda_{j}\right)}{K^{\prime}\left(\lambda_{j}\right)}, \quad\left(\phi_{1 j}+\phi_{2 j}\right)^{2}=2 \frac{\widetilde{R}\left(\lambda_{j}\right)}{K^{\prime}\left(\lambda_{j}\right)}, \quad 1 \leq j \leq N,
$$


which leads to

$$
\left(\phi_{1 j}+\phi_{2 j}\right)=\sqrt{\frac{2 \widetilde{R}\left(\lambda_{j}\right)}{K^{\prime}\left(\lambda_{j}\right)}}, \quad\left(\psi_{2 j}-\psi_{1 j}\right)=\frac{\sqrt{2} \bar{R}\left(\lambda_{j}\right)}{\sqrt{\widetilde{R}\left(\lambda_{j}\right) K^{\prime}\left(\lambda_{j}\right)}}, \quad i \leq j \leq N
$$

By substituting $u_{k}$ into $\bar{A}(\lambda)$ of (4.1), $u_{N+k}$ into $\widetilde{A}(\lambda)$ of (4.2) and noting

$$
(A(\lambda)-B(\lambda))^{2}-\bar{B}(\lambda) B(\lambda)=A^{2}(\lambda)+B(\lambda) C(\lambda)=P(\lambda),
$$

one gets the separated equations $(1 \leq k \leq N)$

$$
\begin{aligned}
& v_{k}=B\left(u_{k}\right)-A\left(u_{k}\right)-\tilde{A}\left(u_{k}\right)=\sqrt{P\left(u_{k}\right)}-\tilde{A}\left(u_{k}\right) \\
& =\sqrt{1+\sum_{j=1}^{N}\left[\frac{P_{j}}{u_{k}-\lambda_{j}}+\frac{P_{N+j}^{2}}{\left(u_{k}-\lambda_{j}\right)^{2}}\right]}-\sum_{j=1}^{N} \frac{P_{N+j}}{u_{k}-\lambda_{j}}, \\
& v_{N+k}=\widetilde{A}\left(u_{N+k}\right)=\sum_{j=1}^{N} \frac{P_{N+j}}{u_{N+k}-\lambda_{j}} \text {. }
\end{aligned}
$$

Replacing $v_{k}$ by the partial derivative $\partial S / \partial u_{k}$ of the generating function $S$ of canonical transformation and interpreting $P_{j}$ and $P_{N+j}$ as integration constants, the above separated equations give rise to the Hamilton-Jacobi equations which are completely separated and can be integrated to give the completely separated solution for $S$

$$
\begin{aligned}
S\left(u_{1}, \ldots, u_{2 N}\right) & =\sum_{k=1}^{N}\left[\int^{u_{k}}(\sqrt{P(\lambda)}-\widetilde{A}(\lambda)) d \lambda+\int^{u_{N+k}} \tilde{A}(\lambda) d \lambda\right] \\
& =\sum_{k=1}^{N}\left[\int^{u_{k}} \sqrt{P(\lambda)} d \lambda-\sum_{j=1}^{N} P_{N+j} \ln \left|\frac{u_{k}-\lambda_{j}}{u_{N+k}-\lambda_{j}}\right|\right] .
\end{aligned}
$$

The linearizing coordinates are then

$$
\left\{\begin{array}{c}
Q_{j}=\frac{\partial S}{\partial P_{j}}=\frac{1}{2} \sum_{k=1}^{N} \int^{u_{k}} \frac{1}{\left(\lambda-\lambda_{j}\right) \sqrt{P(\lambda)}} d \lambda, \\
Q_{N+j}=\frac{\partial S}{\partial P_{N+j}}=\sum_{k=1}^{N}\left[\int^{u_{k}} \frac{P_{N+j}}{\left(\lambda-\lambda_{j}\right)^{2} \sqrt{P(\lambda)}} d \lambda-\ln \left|\frac{u_{k}-\lambda_{j}}{u_{N+k}-\lambda_{j}}\right|\right],
\end{array}\right.
$$

where $1 \leq j \leq N$. These coordinates $Q_{j}$ and $Q_{N+j}$ constitute the action-angle variables together with $P_{j}$ and $P_{N+j}, 1 \leq j \leq N$. By using (3.9) and (3.10), the 
linear flows induced by the $x$-FDIHS (3.7) and the $t_{2}$-FDIHS (3.8) lead to the Jacobi inversion problem for the $x$-FDIHS (3.7)

$$
\left\{\begin{aligned}
2 Q_{j} & =\gamma_{j}+\left(2 \lambda_{j}-\sum_{k=1}^{N} P_{k}\right) x \\
Q_{N+j} & =\gamma_{N+j}+2 P_{N+j} x
\end{aligned}\right.
$$

and the Jacobi inversion problem for the $t_{2}$-FDIHS (3.8)

$$
\left\{\begin{array}{l}
2 Q_{j}=\bar{\gamma}_{j}+\left[2 \lambda_{j}^{2}-\sum_{k=1}^{N}\left(\lambda_{k} P_{k}+\lambda_{j} P_{k}+P_{N+k}^{2}\right)+\frac{3}{4}\left(\sum_{k=1}^{N} P_{k}\right)^{2}\right] t_{2}, \\
Q_{N+j}=\bar{\gamma}_{N+j}+P_{N+j}\left(4 \lambda_{j}-\sum_{k=1}^{N} P_{k}\right) t_{2},
\end{array}\right.
$$

where $1 \leq j \leq N, Q_{j}$ and $Q_{N+j}$ are defined by (4.6), and $\gamma_{j}$ and $\bar{\gamma}_{j}, 1 \leq j \leq 2 N$, are arbitrary constants.

Since the AKNS equations (3.4) are factorized by the $x$-FDIHS (3.7) and the $t_{2}$ FDIHS (3.8), combining the Jacobi inversion problems (4.7) and (4.8) gives rise to the following theorem.

THEOREM 4.2. The AKNS equations (3.4) lead to the Jacobi inversion problem determined by

$$
\begin{aligned}
& \sum_{k=1}^{N} \int^{u_{k}} \frac{1}{\left(\lambda-\lambda_{j}\right) \sqrt{P(\lambda)}} d \lambda \\
& =\tilde{\gamma}_{j}+\left(2 \lambda_{j}-\sum_{k=1}^{N} P_{k}\right) x \\
& +\left[2 \lambda_{j}^{2}-\sum_{k=1}^{N}\left(\lambda_{k} P_{k}+\lambda_{j} P_{k}+P_{N+k}^{2}\right)+\frac{3}{4}\left(\sum_{k=1}^{N} P_{k}\right)^{2}\right] t_{2}, \\
& \sum_{k=1}^{N}\left[\int^{u_{k}} \frac{P_{N+j}}{\left(\lambda-\lambda_{j}\right)^{2} \sqrt{P(\lambda)}} d \lambda-\ln \left|\frac{u_{k}-\lambda_{j}}{u_{N+k}-\lambda_{j}}\right|\right] \\
& =\tilde{\gamma}_{N+j}+2 P_{N+j} x+P_{N+j}\left(4 \lambda_{j}-\sum_{k=1}^{N} P_{k}\right) t_{2},
\end{aligned}
$$

where $1 \leq j \leq N$, and $\tilde{\gamma}_{j}$ and $\tilde{\gamma}_{N+j}, 1 \leq j \leq N$, are arbitrary constants.

We remark that the above Jacobi inversion problem for the AKNS equations (3.4) is different to that in [18], which was generated from another class of canonical separated 
variables for the binary constrained flows (3.7) and (3.8). The above manipulation may also be similarly applied to the whole AKNS hierarchy, and the approach depicted in Section 2 can be applied to other soliton hierarchies such as the KdV hierarchy and the Kaup-Newell hierarchy.

\section{Acknowledgments}

This paper is dedicated to Martin Kruskal's seventy-fifth birthday. This work was in part supported by the City University of Hong Kong (SRGs: 7000945 and 7001041), the Research Grants Council of Hong Kong (CERGs: 9040375 and 9040466) and the Chinese Basic Research Project "Nonlinear Science". One of the authors (Ma) is also grateful to the organizer Nalini Joshi for inviting him to speak at the Kruskal 2000 workshop in Adelaide.

\section{References}

[1] M. J. Ablowitz, D. J. Kaup, A. C. Newell and H. Segur, "The inverse scattering transform-Fourier analysis for nonlinear problems", Studies in Appl. Math. 53 (1974) 249-315.

[2] M. Antonowicz and S. Rauch-Wojciechowski, "Soliton hierarchies with sources and Lax representation for restricted flows", Inverse Problems 9 (1993) 201-215.

[3] B. A. Dubrovin, "Theta-functions and nonlinear equations", Russian Math. Survey 36 (1981) 11-92.

[4] J. C. Eilbeck, V. Z. Enol'skii, V. B. Kuznetsov and A. V. Tsiganov, "Linear $r$-matrix algebra for classical separable systems", J. Phys. A: Math. Gen. 27 (1994) 567-578.

[5] J. Harnad and P. Winternitz, "Classical and quantum integrable systems in $\tilde{\mathbf{g l}}(2)^{+*}$ and separation of variables", Commun. Math. Phys. 172 (1995) 263-285.

[6] P. P. Kulish, S. Rauch-Wojciechowski and A. V. Tsiganov, "Stationary problems for equation of the KdV type and dynamical $r$-matrices", J. Math. Phys. 37 (1996) 3463-3482.

[7] V. B. Kuznetsov, "Quadrics on real Riemannian spaces of constant curvature: separation of variables and connection with Gaudin magnet", J. Math. Phys. 33 (1992) 3240-3254.

[8] Y. Li and W. X. Ma, "Binary linearization of AKNS spectral problem under higher-order symmetry constraints", Chaos, Solitons and Fractals 11 (2000) 697-710.

[9] W. X. Ma, "New finite-dimensional integrable systems by symmetry constraint of the KdV equations", J. Phys. Soc. Jpn. 64 (1995) 1085-1091.

[10] W. X. Ma, "Symmetry constraint of MKdV equations by binary nonlinearization", Physica A219 (1995) 467-481.

[11] W. X. Ma and B. Fuchssteiner, "Binary linearization of the Lax pairs", in Nonlinear Physics (eds. E. Alfinito, M. Boiti, L. Martina and F. Pempinelli), (World Scientific, Singapore, 1996) 217-224.

[12] W. X. Ma, B. Fuchssteiner and W. Oevel, "A $3 \times 3$ matrix spectral problem for AKNS hierarchy and its binary nonlinearization", Physica A233 (1996) 331-354.

[13] W. X. Ma and W. Strampp, "An explicit symmetry constraint for the Lax pairs and the adjoint Lax pairs of AKNS systems", Phys. Lett. A185 (1994) 277-286.

[14] E. K. Sklyanin, "Separation of variables-new trends. Quantum field theory, integrable models and beyond", Prog. Theor. Phys. Suppl. 118 (1995) 35-60. 
[15] Y. Zeng, "Separation of variables for the constrained flows", J. Math. Phys. 38 (1997) 321-329.

[16] Y. Zeng, "Using factorization to solve soliton equations", J. Phys. A. Math. Gen. 30 (1997) 3719-3724.

[17] $\mathrm{Y}$. Zeng and $\mathrm{Y}$. $\mathrm{Li}$, "The deduction of the Lax representation for constrained flows from the adjoint representation", J. Phys. A: Math. Gen. 26 (1993) L273-L278.

[18] Y. Zeng and W. X. Ma, "Separation of variables for soliton equations via their binary constrained flows", J. Math. Phys. 40 (1999) 6526-6557. 